\title{
EFEITO DE INJÚRIAS MECÂNICAS NO PROCESSO RESPIRATÓRIO E NOS PARÂMETROS QUÍMICOS DE GOIABAS 'PALUMA' E 'PEDRO SATO'
}

\author{
BEN-HUR MATTIUZ² \& JOSÉ FERNANDO DURIGAN ${ }^{3}$
}

\begin{abstract}
RESUMO - O objetivo deste trabalho foi avaliar o efeito das injúrias mecânicas por impacto, compressão ou corte na atividade respiratória e na composição química de goiabas 'Paluma' e 'Pedro Sato' colhidas no estádio de maturação "de vez" e armazenadas a $23,4 \pm 1^{\circ} \mathrm{C}$ e $62 \pm 6 \%$ UR (condições de ambiente). Na injúria por impacto, os frutos foram deixados cair, por duas vezes e em queda livre, de uma altura de $1,20 \mathrm{~m}$, sofrendo os impactos em lados opostos de sua porção equatorial. Na injúria por compressão, os frutos foram submetidos a um peso de $3 \mathrm{~kg}$, por 15 minutos. Para a injúria por corte foram efetuados 2 cortes no sentido longitudinal dos frutos, de $30 \mathrm{~mm}$ de comprimento por $2 \mathrm{~mm}$ de profundidade. Os frutos injuriados foram colocados em bandejas de isopor e armazenados sob as condições de ambiente, com a atividade respiratória avaliada diariamente e seus conteúdos de sólidos solúveis totais, açúcares redutores, acidez total titulável e ácido ascórbico, determinados a cada 2 dias. Houve um incremento na evolução da atividade respiratória dos frutos em todos os tratamentos durante o armazenamento, com os injuriados produzindo maior quantidade de $\mathrm{CO}_{2}$ $\left(184,12 \mathrm{mg} \mathrm{de} \mathrm{CO}_{2} \cdot \mathrm{kg}^{-1} \cdot \mathrm{h}^{-1}\right)$ que os do controle $\left(164,23 \mathrm{mg} \mathrm{de} \mathrm{CO}_{2} \cdot \mathrm{kg}^{-1} \cdot \mathrm{h}^{-1}\right)$. Os frutos da 'Paluma' apresentaram essa atividade mais intensa $\left(178,39 \mathrm{mg}\right.$ de $\left.\mathrm{CO}_{2} \cdot \mathrm{kg}^{-1} \cdot \mathrm{h}^{-1}\right)$ que os da 'Pedro Sato' $\left(169,35 \mathrm{mg}\right.$ de $\left.\mathrm{CO}_{2} \cdot \mathrm{kg}^{-1} \cdot \mathrm{h}^{-1}\right)$, e não se detectou durante o período de armazenamento a ocorrência de pico respiratório. Os teores de sólidos solúveis totais $\left(9,39{ }^{\circ}\right.$ Brix $)$, açúcares redutores $(2,24 \mathrm{~g}$ de glicose. $\left.100 \mathrm{~g}^{-1}\right)$, acidez total titulável $\left(706,25 \mathrm{mg}\right.$ de ácido cítrico. $\left.100 \mathrm{~g}^{-1}\right)$ e ácido ascórbico $\left(65,33 \mathrm{mg} .100 \mathrm{~g}^{-1}\right)$ dos frutos injuriados apresentaram-se com valores menores que os do controle, ao longo do período de armazenamento.
\end{abstract}

Termos para indexação: Psidium guajava, pós-colheita, impacto, compressão, corte, respiração, composição química.

\section{EFFECT OF MECHANICAL INJURIES ON RESPIRATORY PROCESS AND CHEMICALS PARAMETERS OF 'PALUMA' AND 'PEDRO SATO' GUAVAS}

\begin{abstract}
This work aimed to evaluate the effect of mechanical injury, impact, compression or cut, on respiratory activity and chemical composition of fruits of two guava cultivars, harvested at mature-green stage and stored to $23.4 \pm 1{ }^{\circ} \mathrm{C}$ and $62 \pm 6 \% \mathrm{RH}$ (laboratory conditions). To produce impact injury, fruits were dropped, twice, from a height of $1.20 \mathrm{~m}$ against a hard surface. The compression injury was produced in fruits submitted to the action of a weight $(3 \mathrm{~kg})$ for 15 minutes. Cut injury was obtained by cutting fruits, twice, in the longitudinal direction, with exactly $30 \mathrm{~mm}$ length and two $\mathrm{mm}$ depth. The fruits were packed in trays and stored under laboratory conditions. The respiratory activity was evaluated daily and chemical composition every two days (total soluble solids, reducing sugars, total titratable acidity and ascorbic acid). There was an increase in respiratory activity of the fruits during the storage, with injured producing more $\mathrm{CO}_{2}\left(184.12 \mathrm{mg}\right.$ of $\left.\mathrm{CO}_{2} \cdot \mathrm{kg}^{-1} \cdot \mathrm{h}^{-1}\right)$ than the control $\left(164.23 \mathrm{mg}\right.$ of $\left.\mathrm{CO}_{2} \cdot \mathrm{kg}^{-1} \cdot \mathrm{h}^{-1}\right)$, for both cultivars. 'Paluma' fruits presented respiratory activity $\left(178.39 \mathrm{mg}\right.$ of $\left.\mathrm{CO}_{2} \cdot \mathrm{kg}^{-1} \cdot \mathrm{h}^{-1}\right)$ higher than 'Pedro Sato' $\left(169.35 \mathrm{mg} \mathrm{of} \mathrm{CO}_{2} \cdot \mathrm{kg}^{-1} \cdot \mathrm{h}^{-1}\right)$, and climacteric peak was not detected. The total soluble solids $\left(9.39^{\circ} \mathrm{Brix}\right)$, reducing sugars $\left(2.24 \mathrm{~g}\right.$ of glucose. $\left.100 \mathrm{~g} \mathrm{~g}^{-1}\right)$, total titratable acidity (706.25 mg of citric acid. $\left.100 \mathrm{~g}^{-1}\right)$ and ascorbic acid (65.33 mg of ascorbic acid. $\left.100 \mathrm{~g}^{-1}\right)$ contents in injured fruits were smaller than in control, along the storage period.
\end{abstract}

Index Terms: Psidium guajava, postharvest, impact, compression, cut, respiration, chemical composition.

\section{INTRODUÇÃO}

O Brasil é o segundo produtor mundial de goiaba (Psidium guajava L.), com uma produção anual de 257 mil toneladas. Apesar da maior parte da produção desta fruta destinarse à industria, no período de 1998-99 houve um incremento de $79 \%$ na comercialização de goiaba vermelha destinada ao consumo como fruta fresca, no CEAGESP - SP (WWWGH, 2000; AGRIANUAL, 2001).

As injúrias mecânicas podem ser definidas como deformações plásticas, rupturas superficiais e destruição dos tecidos vegetais, provocadas por forças externas e que levam a modificações físicas (danos físicos) e/ou alterações fisiológicas, químicas e bioquímicas na cor, aroma, sabor e textura (Mohsenin, 1986).

Estas podem ser agrupadas em injúrias por compressão, impacto ou corte. A injúria de impacto é geralmente causada pela colisão do fruto contra superfícies sólidas ou outros frutos durante as etapas de colheita, manuseio e transporte. A injúria mecânica por compressão é causada pela imposição de uma

1 Trabalho ${ }^{0}$ 266/2000. Recebido: 08/12/2000. Aceito para publicação: 02/08/2001.

2 Doutorando do Curso de Pós-Graduação em Produção Vegetal, FCAV-UNESP. Bolsista da FAPESP. bmattiuz@ig.com.br

3 Prof. Titular do Departamento de Tecnologia, FCAV-UNESP. jfduri@fcav.unesp.br

UNESP - CÂMPUS DE JABOTICABAL, Via de acesso Prof. Paulo Donato Castellane, km 5, 14870-000 
pressão variável contra a superfície externa do fruto, quer seja por um fruto adjacente ou pela própria parede da embalagem em que está acondicionado o produto. A injúria por corte é geralmente atribuída à colisão da superfície fruto contra uma muito menor que a primeira ocasionando a ruptura da epiderme, ou pela imposição de uma pressão sobre o fruto contra superfícies também desiguais, como as arestas de uma embalagem. Existem informações indicando que tais injúrias ocasionam danos irreparáveis em frutos como cerejas (Burton \& Schulte-Pason, 1987), tangerinas 'Satsuma'(Iwamoto et al., 1984), melões 'Cantaloupe' (MacLeod et al., 1976) e maçãs (Parker et al., 1984), provocando aumentos na atividade respiratória e alterações químicas, reduzindo sua vida útil.

O objetivo deste trabalho foi avaliar o efeito das injúrias mecânicas por impacto, compressão ou corte na atividade respiratória e na composição química de goiabas 'Paluma' e 'Pedro Sato', armazenadas sob condições de ambiente.

\section{MATERIAL E MÉTODOS}

Foram utilizados frutos de goiabeiras das cultivares Paluma e Pedro Sato procedentes de propriedade particular, distante $43 \mathrm{~km}$ do local de condução dos experimentos, colhidos em duas épocas: janeiro (experimento de impacto) e outubro de 1999 (experimentos de compressão e corte).

Depois de colhidos, no estádio de maturação "de vez", correspondente a coloração verde-mate (Pereira, 1995), os frutos foram imediata e cuidadosamente transportados para o Laboratório de Tecnologia dos Produtos Agrícolas da FCAV/ UNESP - Jaboticabal, SP.

Após imersão em água fria $\left(15^{\circ} \mathrm{C}\right)$ e clorada $(150 \mu \mathrm{g}$ de cloro. $\left.\mathrm{L}^{-1}\right)$ por 5 minutos, estes frutos foram submetidos às injúrias mecânicas. Na injúria por impacto, eles foram deixados cair, em queda livre, de uma altura de 1,20 m. Cada fruto sofreu dois impactos na região equatorial, em lados opostos. Para a injúria correspondente a compressão, os frutos foram colocados sob um aparelho onde um bloco exercendo um peso de $3 \mathrm{~kg}$ era apoiado, por $15 \mathrm{~min}$. Na injúria por corte foram realizados 2 cortes de $30 \mathrm{~mm}$ de comprimento por $2 \mathrm{~mm}$ de profundidade, no sentido longitudinal dos frutos, usando-se uma lâmina com 1,1 $\mathrm{mm}$ de espessura.

As áreas lesionadas foram demarcadas e os frutos acondicionados, em bandejas de isopor e armazenados sob condições de ambiente $\left(23,4 \pm 1{ }^{\circ} \mathrm{C}\right.$ e $62 \pm 6 \%$ UR).

A produção de $\mathrm{CO}_{2}$ foi determinada diariamente, colocando-se quatro frutos em um recipiente com capacidade de 3 litros, hermeticamente fechado, por um período de 1 hora, sob as condições de ambiente. O conteúdo de $\mathrm{CO}_{2}$ da atmosfera do interior do recipiente era determinado, antes e imediatamente após este período, em cromatógrafo GC Finnigan 9001.

Para análises químicas as amostras eram tomadas a cada dois dias utilizando-se somente o pericarpo dos frutos injuriados, que depois de se separar a área lesionada da não lesionada, era triturado até a homogeneização e mantido $\mathrm{a}-18^{\circ} \mathrm{C}$, até a análise, efetuada na semana seguinte. Estas amostras foram analisadas quanto aos seus conteúdos de ácido ascórbico, acidez total titulável, sólidos solúveis totais (AOAC, 1980) e açúcares redutores (Villela et al., 1973).

\section{RESULTADOS E DISCUSSÃO}

Os frutos das duas cultivares apresentaram incremento na evolução da atividade respiratória, durante o período de armazenamento (Tabela 1) a qual também foi detectada por Mercado-Silva et al. (1998), no México, em goiabas da cultivar Media China, colhidas na primavera-verão, onde apresentaram produção de $30 \mathrm{mg}$ de $\mathrm{CO}_{2} \cdot \mathrm{kg}^{-1} \cdot \mathrm{h}^{-1}$ por ocasião da colheita e de $200 \mathrm{mg}$ de $\mathrm{CO}_{2} \cdot \mathrm{kg}^{-1} \cdot \mathrm{h}^{-1}$, cinco dias após.

Durante o período de armazenamento, os frutos injuriados, de ambas as cultivares, produziram uma quantidade maior de $\mathrm{CO}_{2}$ que os não injuriados (Tabela 1). Esses resultados reafirmam aqueles encontrados por Burton \& Schulte-Pason (1987), que evidenciaram um aumento na evolução de $\mathrm{CO}_{2}$ de cerejas submetidas a várias intensidades de impacto. Estes autores atribuíram esse incremento à descarboxilação do ácido málico, que teria extravasado das células danificadas no local de ocorrência das injúrias. Na injúria por impacto, os frutos lesionados apresentaram incrementos maiores na produção de $\mathrm{CO}_{2}$ que nas demais injúrias. Isto evidencia que esta lesão é grave, provavelmente devido a um maior rompimento de células por ocasião da injúria. Injúrias por impacto também foram responsáveis por aumentos na atividade respiratória de melões Cantaloupe (MacLeod et al., 1976) e de maçãs (Parker et al., 1984).

Ainda na Tabela 1, tem-se que não houve a ocorrência de um pico respiratório durante o período de avaliação. A definição do comportamento respiratório pós-colheita desta fruta ainda é objeto de dúvida, pois enquanto Durigan (1997) relata que trabalhos antigos classificam esta fruta como possuindo um comportamento não climatérico, outros autores têm demonstrado que seu comportamento é climatérico (Mercado-Silva et al., 1998). Estes autores demonstraram que a estação do ano interfere nesse pico, ou seja, goiabas colhidas no período mais frio (outono-inverno) exibiam uma maior produção de $\mathrm{CO}_{2}$ no quinto dia após a colheita, enquanto que goiabas colhidas na primaveraverão atingiam este pico no oitavo dia. Neste experimento esse pico não foi evidenciado até o sétimo dia, quando os frutos já se encontravam em fase adiantada de senescência. Informações mais recentes revelam que a definição do comportamento fisiológico da goiaba está extremamente vinculada à cultivar (comunicação pessoal do Dr. Edmundo Mercado-Silva, professor da Facultad de Química, Universidad Autónoma de Queretaro, México, 2000).

Verifica-se, através da Tabela 2, que o conteúdo de sólidos solúveis totais manteve-se estável durante o período de armazenamento. No entanto, observa-se que nos frutos injuriados houve uma tendência a valores menores que nos frutos controle, independentemente da cultivar e do tipo de injúria aplicada. Notase também que as menores médias foram obtidas na injúria por impacto, para ambas as cultivares, o que leva frutos submetidos a este tipo de injúria a uma maior perda na qualidade.

$\mathrm{Na}$ Tabela 3 são mostrados os dados referentes ao efeito das injúrias mecânicas sobre o conteúdo de açúcares redutores, os quais, apesar da não ocorrência de diferenças significativas do efeito das injúrias durante o período de armazenamento, apresentou tendência de decréscimo ao longo do período de armazenamento, o que pode ser devido ao aumento na atividade respiratória. Sharaf \& El-Saadany (1986) também observaram 
redução nos teores de açúcares redutores em goiabas durante a evolução do estádio de maturação verde maduro a completamente maduro. Observa-se também, que os frutos injuriados apresentaram valores menores que os do controle, independentemente da cultivar. A redução do conteúdo de açúcares redutores e sólidos solúveis totais (Tabela 2) nos frutos injuriados pode estar relacionada à utilização destes compostos como substrato respiratório, visto que nesses frutos houve um maior incremento na produção de $\mathrm{CO}_{2}$ que em frutos intactos (Tabela 1).

Na Tabela 4 são apresentados os resultados do efeito dos três tipos de injúrias sobre a acidez total titulável das goiabas, durante o período de armazenamento sob condições de ambiente. Verifica-se que a evolução apresentou aumento nos teores de ácido cítrico até o quarto dia após a injúria, com decréscimo a seguir, independentemente do efeito da cultivar e da injúria. Nos frutos injuriados observaram-se menores teores desse ácido que nos controle, ao longo do período de avaliação. O aumento da atividade respiratória pode ter desencadeado o incremento da produção de ácido cítrico, via ciclo de Krebs, até o quarto dia, sendo consumido a seguir como substrato respiratório. O observado vem ao encontro do trabalho de Moretti (1998) no qual se observou que a injúria mecânica por impacto levou a reduções na acidez titulável de tomates, a qual foi atribuída a diminuição da concentração de ácidos orgânicos.

A evolução do teor de ácido ascórbico na polpa das goiabas pode ser observada na Tabela 5, a qual não foi significativa. Os valores detectados nos frutos injuriados, ao longo do período, são menores que nos controle sendo que lesões de impacto e compressão levaram a valores médios menores. Provavelmente, os sistemas protetores antioxidantes associados ao ácido ascórbico são danificados pela injúria mecânica, permitindo a depleção oxidativa irreversível do ácido ascórbico a ácido 2,3 dioxi L-gulônico (Burton, 1982).

TABELA 1. Atividade respiratória ( $\left.\mathrm{mg} \mathrm{de} \mathrm{CO}_{2} \cdot \mathrm{kg}^{-1} \cdot \mathrm{h}^{-1}\right)$ de goiabas 'Paluma' e 'Pedro Sato', submetidas ou não, a três tipos de injúria mecânica e armazenadas sob condições de ambiente $\left(23,4 \pm 1{ }^{\circ} \mathrm{C}\right.$ e $62 \pm 6 \%$ UR).

\begin{tabular}{|c|c|c|c|c|c|}
\hline \multirow{2}{*}{$\begin{array}{c}\mathrm{T} \text { e m p o } \\
\text { (d i a ) }\end{array}$} & \multicolumn{5}{|c|}{ A tividade respiratória } \\
\hline & \multicolumn{2}{|c|}{$\mathrm{Im} \mathrm{p} \mathrm{a} \mathrm{c} \mathrm{to}$} & \multicolumn{2}{|c|}{ Co m pressão } & Corte \\
\hline \multicolumn{6}{|c|}{ Cultivar Paluma } \\
\hline & 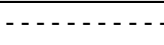 & $-\cdots$ & $-\mathrm{Contro}$ & --- & ( \\
\hline 1 & 160,14 & $\mathrm{c}$ & 104,58 & $\mathrm{e}$ & 120,10 \\
\hline 2 & 194,41 & $\mathrm{c}$ & 123,89 & $\mathrm{~d}$ & 134,44 \\
\hline 3 & 174,84 & $\mathrm{c}$ & 139,18 & $\mathrm{c} \mathrm{d}$ & 154,17 \\
\hline 5 & 273,34 & $\mathrm{a} b$ & 168,07 & $\mathrm{ab}$ & 217,57 \\
\hline \multirow[t]{2}{*}{6} & 325,37 & a & 176,30 & $\mathrm{a}$ & 246,56 \\
\hline & $\ldots$ & $-\ldots$ & In juriac & a - - & ( \\
\hline 0 & 186,22 & $\mathrm{c}$ & 112,09 & $\mathrm{~d}$ & 121,50 \\
\hline 1 & 180,07 & $\mathrm{c}$ & 121,09 & $\mathrm{c} \mathrm{d}$ & 122,89 \\
\hline 2 & 218,59 & $\mathrm{c}$ & 139,27 & $\mathrm{c}$ & 139,60 \\
\hline M éd ia & 228,20 & A & 145,72 & $\mathrm{C}$ & 174,37 \\
\hline C.V. $(\%)$ & \multicolumn{2}{|c|}{27,93} & 22,7 & & 32,40 \\
\hline \multicolumn{6}{|c|}{ Cultivar Pedro Sato } \\
\hline \multicolumn{6}{|c|}{ - } \\
\hline 0 & 153,50 & $\mathrm{~d}$ & 83,69 & $\mathrm{~d}$ & 119,26 \\
\hline 1 & 155,08 & $\mathrm{~d}$ & 87,70 & $\mathrm{~d}$ & 122,24 \\
\hline 2 & 201,36 & $\mathrm{bccd}$ & 96,47 & $\mathrm{c} \mathrm{d}$ & 137,31 \\
\hline 3 & 185,50 & $\mathrm{c} \mathrm{d}$ & 110,06 & $\mathrm{bcc}$ & 140,79 \\
\hline 4 & 212,57 & $\mathrm{a} b \mathrm{c}$ & 124,40 & $\mathrm{~b}$ & 158,98 \\
\hline 5 & 253,21 & $\mathrm{a} b$ & 147,30 & $\mathrm{a}$ & 180,17 \\
\hline 6 & 261,39 & $\mathrm{a}$ & 151,36 & $\mathrm{a}$ & 195,60 \\
\hline M éd ia & 215,09 & $\mathrm{~A}$ & 120,62 & $\mathrm{C}$ & 161,04 \\
\hline C.V. $(\%)$ & 20,38 & & 24,7 & & 26,14 \\
\hline
\end{tabular}

* Para cada cultivar, médias seguidas por uma mesma letra, maiúscula na linha e minúscula na coluna, não diferem significativamente entre si, pelo teste de Tukey ( $\mathrm{P}<0,05)$. 
TABELA 2. Conteúdo de sólidos solúveis totais ( ${ }^{\circ}$ Brix) em goiabas 'Paluma' e 'Pedro Sato', submetidas ou não, a três tipos de injúria mecânica e armazenadas sob condições de ambiente $\left(23,4 \pm 1{ }^{\circ} \mathrm{C}\right.$ e $62 \pm 6 \%$ UR).

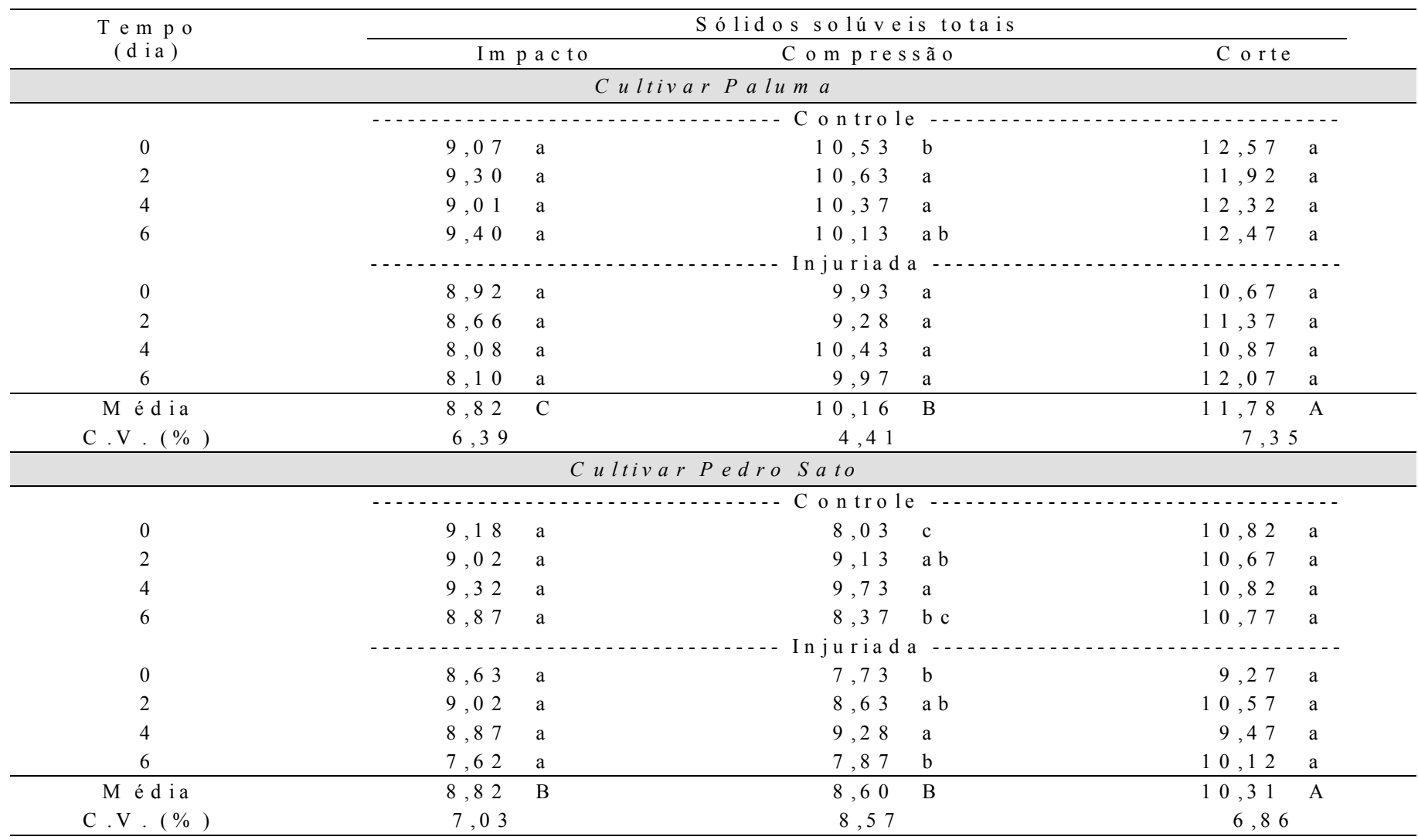

* Para cada cultivar, médias seguidas por uma mesma letra, maiúscula na linha e minúscula na coluna, não diferem significativamente entre si, pelo teste de Tukey (P<0,05).

TABELA 3. Conteúdo de açúcares redutores (g de glicose. $\left.100 \mathrm{~g}^{-1}\right)$ em goiabas 'Paluma' e 'Pedro Sato', submetidas ou não, a três tipos de injúria mecânica e armazenadas sob condições de ambiente $\left(23,4 \pm 1^{\circ} \mathrm{C}\right.$ e $62 \pm 6 \%$ UR).

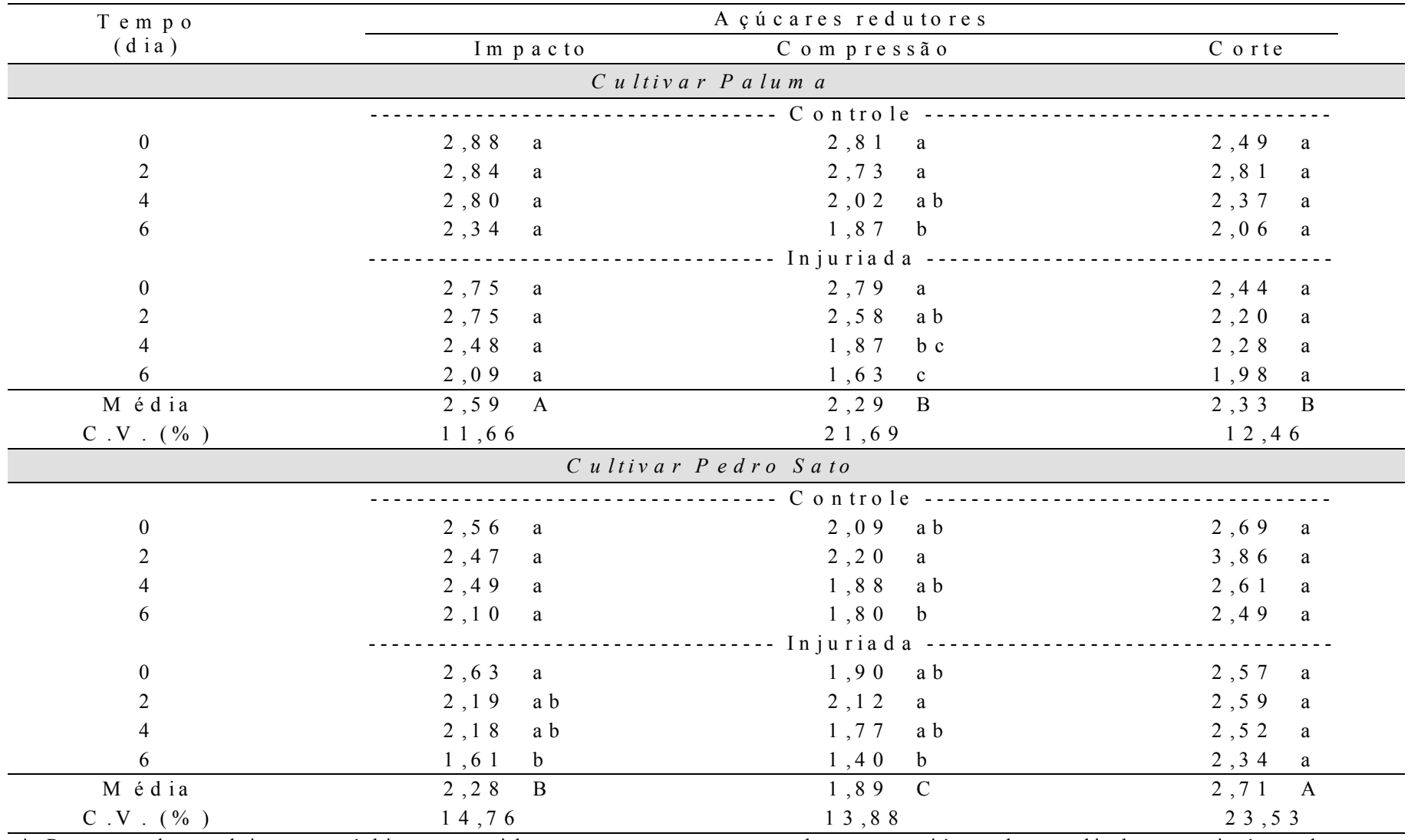

* Parā cada cultivar, médias seguidas por úma mesma letra, maiúscula na linha e minúscula na coluna, não diferem significativamente entre si, pelo teste de Tukey (P<0,05). 
TABELA 4. Conteúdo de acidez total titulável ( $\mathrm{mg}$ de ácido cítrico. $\left.100 \mathrm{~g}^{-1}\right)$ em goiabas 'Paluma' e ‘Pedro Sato', submetidas ou não, a três tipos de injúria mecânica e armazenadas sob condições de ambiente $\left(23,4 \pm 1{ }^{\circ} \mathrm{C}\right.$ e $62 \pm 6 \%$ UR).

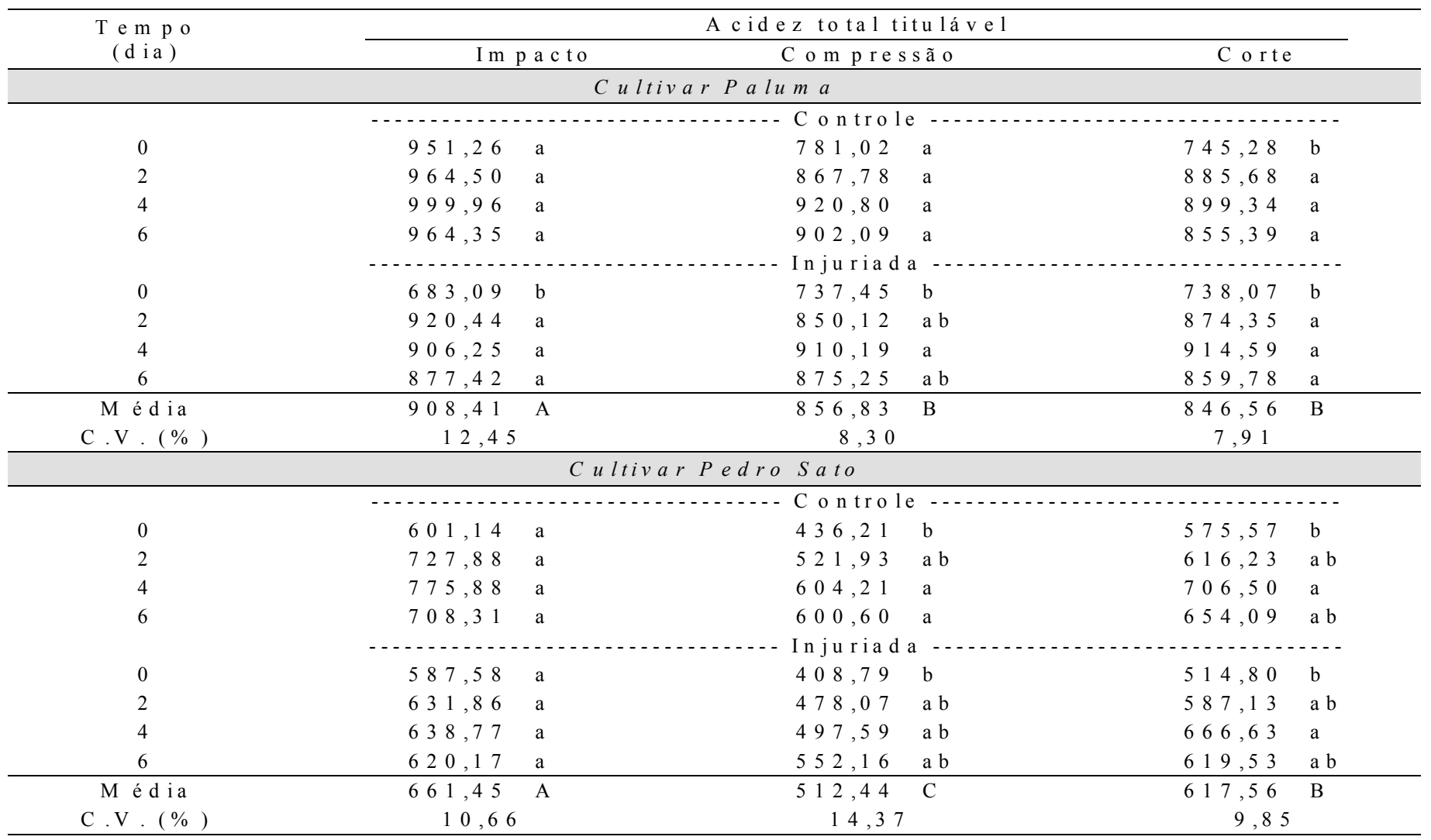

* Para cada cultivar, médias seguidas por uma mesma letra, maiúscula na linha e minúscula na coluna, não diferem significativamente entre si, pelo teste de Tukey (P<0,05).

TABELA 5. Conteúdo de ácido ascórbico (mg. $\left.100 \mathrm{~g}^{-1}\right)$ em goiabas 'Paluma' e 'Pedro Sato', submetidas ou não, a três tipos de injúria mecânica e armazenadas sob condições de ambiente $\left(23,4 \pm 1^{\circ} \mathrm{C}\right.$ e $62 \pm 6$ \% UR).

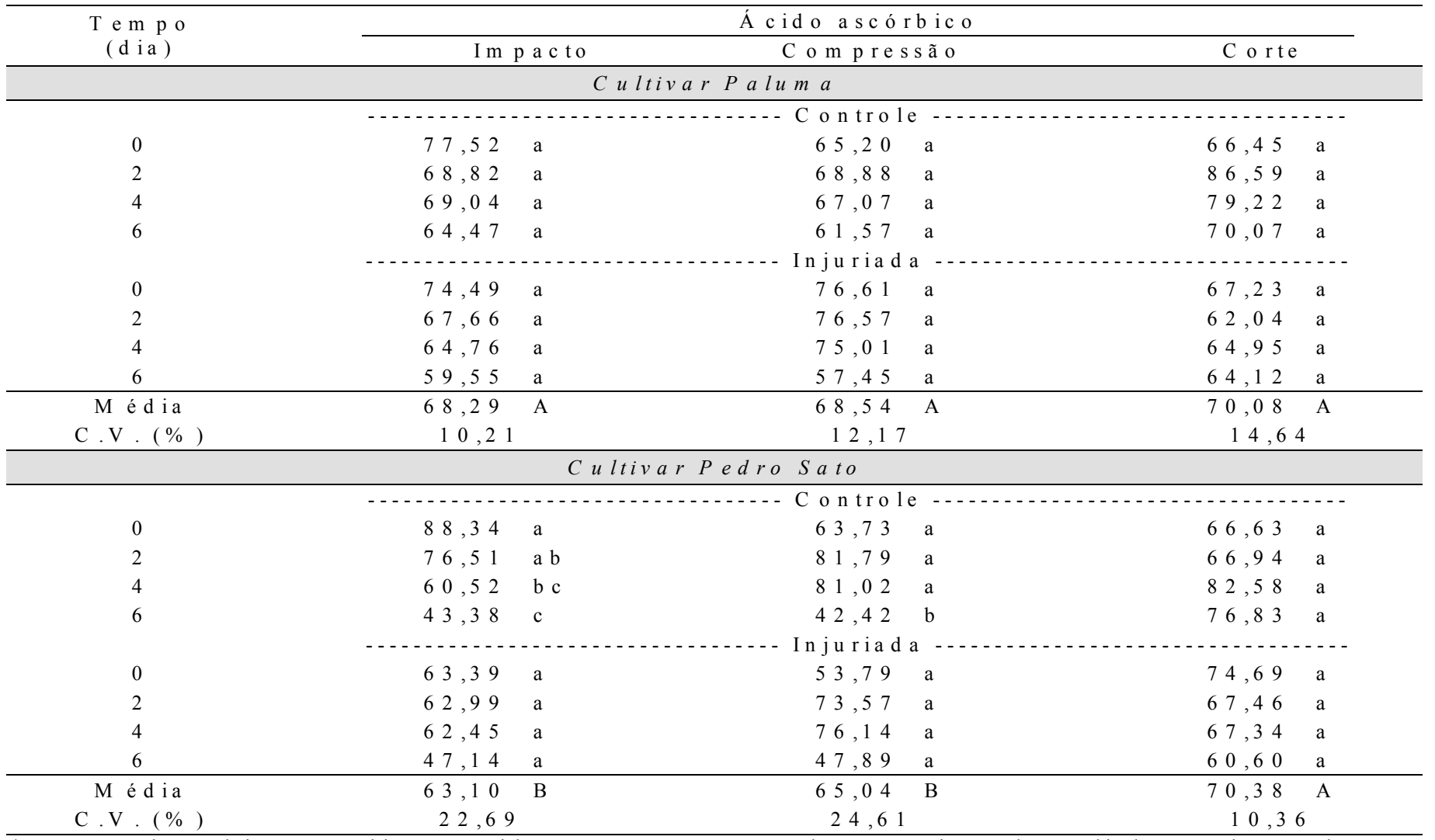

* Para cada cultivar, médias seguidas por úma mesma letra, maiúscula na linha e minúscula na coluna, não diferem significativamente entre si, pelo teste de Tukey $(\mathrm{P}<0,05)$. 


\section{CONCLUSÕES}

Houve um incremento da atividade respiratória ao longo do período de armazenamento, independente das injúrias e das cultivares, não ocorrendo pico respiratório. As injúrias mecânicas promoveram aumento na atividade respiratória de goiabas 'Paluma' e 'Pedro Sato'. Não se detectou a ocorrência de pico respiratório durante o período de armazenamento. Os teores de ácido ascórbico, acidez total titulável, sólidos solúveis totais e açúcares redutores foram menores nos frutos injuriados que nos controle, ao longo do período de armazenamento.

\section{REFERÊNCIAS BIBLIOGRÁFICAS}

AGRIANUAL 2001. Anuário da agricultura brasileira. São Paulo: FNP Consultoria e Comércio. 2001. p. 364-368.

AOAC. Official methods of analysis of the Association of Official Analytical Chemists. 13 ed. Washington: A.O.A.C., 1980. $1018 \mathrm{p}$.

BURTON, C.L.; SCHULTE-PASON, N.L. Carbon dioxide as an indicator of fruit impact damage. HortScience, Alexandria, v. 22, n. 2 , p. $281-2,1987$.

BURTON, W.G. Continue development and changes in quality. In: Postharvest physiology of food crops. New York:Longman, 1982, p. 147-80.

DURIGAN, J.F. Colheita, conservação e embalagens. In: SIMPÓSIO BRASILEIRO SOBRE A CULTURA DA GOIABEIRA, 1, 1997, Jaboaticabal. Anais... Jaboticabal:FACVJ/UNESP-FUNEP-GOIABRÁS, 1997, p. 14958.

IWAMOTO, M.; HAYAKAWA, A.; KAWANO, S.; MANAGO, $M$. Effect of dropping practice in packing house lines on the quality of Satsuma mandarin. Annals of the Engineering Society, v. 45, n. 4, p. 539-44, 1984.
MacLEOD, R.F.; KADER, A.A.; MORRIS, L.L. Damage to fresh tomatoes can be reduced. California Agriculture, Berkeley, v. 30, n. 12, p. 10-12, 1976.

MERCADO-SILVA, E.; BENITO-BAUTISTA, P.; GARCIAVELASCO, M. A. Fruit development, harvest index and ripening changes of guavas produced in central Mexico. Postharvest Biology and Technology, Amsterdam, v. 13, p. 143-50, 1998.

MOHSENIN, N.N. Physical properties of plant and animal material: structure, physical characteristics and mechanical properties. New York: Gordon and Breach, $2^{\mathrm{a}}$ ed., 1986, v. 1, $534 \mathrm{p}$.

MORETTI, C.L. Injúria interna de impacto em frutos de tomate: fisiologia e conservação pós-colheita. Viçosa, 1998. 132 p. Tese (Doutorado em Produção Vegetal), Universidade Federal de Viçosa. Viçosa - MG.

PARKER, M.L.; WARDOWSKI, W.F.; DEWEY, D.H. A damage test for oranges in a commercial packing house line. Proceedings of the Florida State Horticultural Society, Winter Haven, v. 97, p. 136-7. 1984.

PEREIRA, F.M. Cultura da goiabeira. Jaboticabal:FUNEP, $1995.47 \mathrm{p}$.

SHARAF, A.; EL-SAADANY, S.S. Biochemical studies on guava fruits during different maturity stages. Annals of Agricultural Science, Moshtohor, v. 24, n. 2, p. 975-84, 1986.

VILLELA, G., BACILA, M., TASTALDI, H. Técnicas e experimentos de bioquímica. Rio de Janeiro:Guanabara Koogam, 1973. 552 p.

WWWGH. Welcome to the Wonderful World of Guavas Homepage. Distribution. Disponível em <http:// www.ocf.berkeley.edu/ montymex/guava/guavaintro.html $>$. Acessado em 29 de novembro. 2000. 